\title{
Reply on: Comment on: Hellmund et al. Rupture of renal artery aneurysm during late pregnancy: clinical features and diagnosis. Arch Gynecol Obstet. 2016;293:505-8
}

\author{
Astrid Hellmund $^{1} \cdot$ Carsten Meyer $^{1} \cdot$ Ulrich Gembruch $^{1}$
}

Received: 15 April 2016/Accepted: 28 April 2016/Published online: 13 May 2016

(C) Springer-Verlag Berlin Heidelberg 2016

We thank Dr. Greco and colleagues for their interest and adding of valuable information to our paper.

As the authors stated, there is a considerable lack of evidence for the optimal treatment of ruptured renal artery aneurysms during pregnancy. Available data are based on case reports as included in our study. We agree with Dr. Greco and colleagues that whether identified renal artery aneurysms during pregnancy should be treated by surgery or by careful surveillance remains unclear, for the current management of renal artery aneurysms does not include the higher risk of rupture in pregnant women. Moreover, renal artery aneurysms are rarely detected in pregnant women, and the life-threatening event of sudden rupture occurs unpredictably.

We are grateful for adding the results of the large retrospective study of Buck et al., which was published more lately than our paper, illustrating the equal rate for in- hospital mortality regarding 1627 cases of open surgery and 1082 elective endovascular repairs. However, the question whether endovascular repair or open surgery, including heterotopic and orthotopic kidney transplant, should be considered the first line in pregnant women and if the risk of losing the kidney after a trial of endovascular repair is much larger cannot be answered yet, and further studies are mandatory. To our opinion, the first line therapy in cases of ruptured renal artery aneurysms in pregnancy should be dependent on the availability of an experienced team of endovascular surgeons/radiologists in the attending unit and can be considered in cardiovascular stable patients.

\section{Compliance with ethical standards}

Conflict of interest None.

Astrid Hellmund

Astrid.Hellmund@ukb.uni-bonn.de

1 University Hospital Bonn, Bonn, Germany 